\title{
Redislocation after Bearing Exchange for the Treatment of Mobile Bearing Dislocation in Medial Unicompartmental Knee Arthroplasty
}

\author{
Sang-Gyun Kim, $\mathrm{MD}^{1}$, Hyun-Gon Kim, $\mathrm{MD}^{1}$, Seung-Yup Lee, $\mathrm{MD}^{2}$, Hong-Chul Lim, $\mathrm{MD}^{2}$, and Ji-Hoon Bae, $\mathrm{MD}^{1}$ \\ ${ }^{1}$ Department of Orthopaedic Surgery, Korea University Guro Hospital, Korea University College of Medicine, Seoul; ${ }^{2}$ Department of Orthopaedic Surgery, Seoul \\ Barunsesang Hospital, Seoul, Korea
}

\begin{abstract}
Purpose: This study was conducted to investigate the outcomes of bearing exchange for the treatment of mobile bearing dislocation in medial unicompartmental knee arthroplasty (UKA).

Materials and Methods: We retrospectively reviewed 18 patients (15 females and 3 males, mean age of 65 years) treated with bearing exchange following mobile bearing dislocation in medial UKA. The occurrence of bearing redislocation, the Oxford Knee Score, and radiographic changes at the last follow-up were investigated.

Results: Bearing redislocation after bearing exchange occurred in 9 of 18 patients (50\%). Of these 9 patients, 7 underwent conversion to total knee arthroplasty after bearing redislocation. The 9 patients without bearing redislocation showed good to excellent clinical outcomes at a mean followup of 55 months after bearing exchange. The non-redislocation group had a higher percentage of posterior dislocation of the bearing than the redislocation group ( $55.5 \%$ vs. $22.2 \%, \mathrm{p}=0.040$ ). Univariate logistic regression analysis showed no significant risk factors for bearing redislocation. Conclusions: This study showed a high rate of bearing redislocation after isolated, mobile bearing exchange for bearing dislocation following medial UKA. Therefore, bearing exchange as a sole treatment should be carefully considered in selected patients with correctable causes of bearing dislocation.
\end{abstract}

Keywords: Knee, Arthroplasty, Unicompartmental, Dislocation

\section{Introduction}

Bearing dislocation is a specific complication of mobile bearing unicompartmental knee arthroplasty (UKA), and the rate of bearing dislocation in medial UKA ranges from $0.5 \%$ to $5 \%$ in the medical literature ${ }^{1-5}$. Bearing dislocation is mostly caused by surgical technical errors such as flexion/extension gap mismatch,

Received October 27, 2017; Revised (1st) February 11, 2018;

(2nd) May 2, 2018; (3rd) May 2, 2018; Accepted May 4, 2018

Correspondence to: Ji-Hoon Bae, MD

Department of Orthopaedic Surgery, Korea University Guro Hospital, Korea University College of Medicine, 148 Gurodong-ro, Guro-gu, Seoul 08308, Korea

Tel: +82-2-2626-3296, Fax: +82-2-2626-1164

E-mail: osman@korea.ac.kr

This is an Open Access article distributed under the terms of the Creative Commons Attribution Non-Commercial License (http://creativecommons.org/licenses/by-nc/4.0/) which permits unrestricted non-commercial use, distribution, and reproduction in any medium, provided the original work is properly cited. medial collateral ligament (MCL) damage, impingement by remnant cement or osteophytes, and component misalignment ${ }^{6)}$. It can also be caused by progressive polyethylene wear or late component loosening ${ }^{6}$. As most bearing dislocations are symptomatic and uncomfortable, they require prompt surgical management.

The treatment options for bearing dislocation include closed reduction, open exploration and bearing exchange, conversion to fixed UKA, or conversion to total knee arthroplasty (TKA). One of these options can be selected based on the cause of dislocation and the patient's condition. Closed reduction is rarely performed because it is highly unlike to address the underlying cause. Conversion to fixed UKA is another option; however, revision of a failed UKA to another UKA has been less successful than revision from UKA to $\mathrm{TKA}^{7)}$. In general, bearing exchange or conversion to TKA are the preferred treatment options. If there is implant loosening, MCL damage, or a serious mismatch between flexion and extension gaps, conversion to TKA is recommended. When the cause of bearing dislocation is impingement of the 
bearing against retained cement or osteophytes, insertion of a new bearing can be considered after removal of the bone or cement that may be impinging on the bearing if both the femoral and tibial implants are securely fixed with good alignment ${ }^{8}$.

In the literature, we found that many bearing dislocations have been treated with bearing exchange ${ }^{1,4,9-11)}$. Bearing exchange has several advantages, including shorter operation time, shorter hospital stay, faster recovery, maintenance of bone stock, lower patient morbidity, and lower cost. Furthermore, this less invasive surgery might be advantageous in older patients with medical comorbidities. However, there is a risk of bearing redislocation, especially in patients in whom underlying causes are not adequately corrected $^{5,12-14)}$. In addition, when the cause of bearing dislocation is unclear, it is doubtful that bearing exchange can prevent bearing redislocation.

Currently, there is a paucity of studies that have reported the survivorship of bearing exchange and the rate of mobile bearing redislocation in medial UKA. In addition, the proper indications for bearing exchange are not established for the treatment of bearing dislocation, which are mandatory to prevent repeated surgeries. The primary purpose of this study was to investigate the outcomes of bearing exchange for the treatment of mobile bearing dislocation in medial UKA. Furthermore, we investigated whether there were any risk factors for bearing redislocation following bearing exchange.

\section{Materials and Methods}

\section{Study Design}

After obtaining approval from the Institutional Ethical Review Board at our hospital, we retrospectively reviewed the electronic medical records of all patients who underwent a medial mobile UKA between January 2001 and December 2014. We identified 23 patients having a polyethylene bearing dislocation, all of whom were treated with either bearing exchange or conversion to TKA, based on their general health and the causes of dislocation. Patient demographics, clinical data, radiographic data, intraoperative findings at the time of revision surgery for bearing dislocation, and available recent follow-up information were collected from the medical records of enrolled patients. When any relevant data was missing, patients were contacted by telephone to obtain pertinent information about their current status.

\section{Treatment of Bearing Dislocation}

All the primary UKAs were performed using a minimally invasive approach ${ }^{6,15)}$, and Oxford medial partial knee implants (Zim- mer Biomet, Warsaw, IN, USA) were used in all patients. During the revision for bearing dislocation, the same approach was used on the previous surgical scar. After opening the joint, we removed the dislocated bearing and tried to determine the causes of the dislocation. Any structures that might have been impinging on the bearing, implant stability, status of collateral and cruciate ligaments, and flexion/extension gap balance using filler gauges were checked. If there was implant loosening, a serious mismatch between the flexion and extension gap, ligament damage, or misalignment of the implants, the condition was treated with conversion to TKA. When the bearing dislocation was caused by impingement, any bone or remnant cement of the soft tissues that appeared to be impinging was removed and a new bearing was inserted. After revision, postoperative rehabilitation was similar to that administered with primary UKA or TKA.

\section{Outcome Assessments}

The primary outcome was the occurrence of bearing redislocation after bearing exchange. The secondary outcomes were the Oxford Knee Score (excellent, 40-48; good, 30-39; fair, 20-29; and poor, $0-19)^{16)}$, and radiographic changes at the last followup visit in patients without bearing redislocation. During this visit, radiographs displaying a standing anteroposterior view of the lower extremities, standing posteroanterior view with $45^{\circ}$ knee flexion, standing lateral view with $30^{\circ}$ knee flexion, and the Merchant view were taken for assessing any changes in implant position, limb alignment, radiolucency, and progression of osteoarthritis in the lateral compartment. The alignment of the femoral and tibial implants was determined using the postoperative radiograph evaluation manual ${ }^{6)}$.

\section{Statistical Analysis}

Descriptive analyses were reported using means and standard deviations for continuous variables and frequencies and percentages for categorical variables. Pre-, intra-, and postoperative variables were compared between the bearing redislocation group and the non-redislocation group using the Mann-Whitney $U$-test for parametric data and the Fisher exact test for non-parametric data. Normal distribution was assessed using the KolmogorovSmirnov test. Subgroup analysis was performed to evaluate any existing differences between patients with and without bearing redislocation after bearing exchange. Logistic regression analysis was performed to identify the significant risk factors for bearing redislocation. All analyses were performed using SPSS ver. 12.0 (SPSS Inc., Chicago, IL, USA). A p $<0.05$ was considered statistically significant. 


\section{Results}

\section{Patient Demographics at the Time of Initial Bearing Dislocation}

During the study period, the rate of bearing dislocation was 5\% (23/454 UKAs). Of the 23 patients with bearing dislocation, 18 patients were treated with bearing exchange (Table 1). Five patients were excluded from the study as they were treated with conversion to TKA. There were 15 females and 3 males with a mean age of 65 years. The mean time to dislocation of these 18 patients was 41 months (range, 1 to 102 months), and 4 of the dislocations occurred within the first year of surgery. Bearing dislocation occurred under the following circumstances: 13, during daily activities such as rising from a chair or the floor; 3 , following a slip; 1 , due to extensive wear; and 1 , due to a bearing fracture. The direction of dislocation was anterior in 9 patients, posterior in 7 patients, both anterior and posterior in 1 patient (bearing fracture), and medial in 1 patient.

Table 1. Demographics of Patients Treated with Bearing Exchange at the Initial Bearing Dislocation

\begin{tabular}{|c|c|c|c|c|c|c|c|}
\hline No. & Sex & $\begin{array}{l}\text { Age } \\
(\mathrm{yr})\end{array}$ & Diagnosis & $\begin{array}{l}\text { Time to } \\
\text { dislocation } \\
\quad(\mathrm{mo})\end{array}$ & $\begin{array}{l}\text { Direction of } \\
\text { dislocation }\end{array}$ & $\begin{array}{c}\text { Possible causes } \\
\text { of dislocation }\end{array}$ & $\begin{array}{l}\text { Bearing size } \\
\text { at exchange }\end{array}$ \\
\hline \multirow[t]{2}{*}{$1^{\text {a) }}$} & $\mathrm{F}$ & 66 & $\mathrm{OA}$ & 3 & Posterior & Gap imbalance & $3 \rightarrow 4 \rightarrow \mathrm{TKA}$ \\
\hline & & & & 90 & Posterior & Gap imbalance & \\
\hline 2 & $\mathrm{~F}$ & 48 & OA & 27 & Posterior & Gap imbalance & $4 \rightarrow 5$ \\
\hline 3 & $\mathrm{~F}$ & 57 & OA & 29 & Posterior & Component malalignment & $6 \rightarrow 9$ \\
\hline 4 & $\mathrm{~F}$ & 74 & OA & 41 & Posterior & Component malalignment & $3 \rightarrow 5$ \\
\hline 5 & $\mathrm{~F}$ & 76 & OA & 61 & Anterior & MCL laxity & $4 \rightarrow 6$ \\
\hline 6 & $\mathrm{~F}$ & 61 & $\mathrm{OA}$ & 49 & Posterior & Acute trauma & $6 \rightarrow 7$ \\
\hline 7 & $\mathrm{~F}$ & 61 & OA & 15 & Anterior & Gap imbalance & $3 \rightarrow 4$ \\
\hline \multirow[t]{2}{*}{$8^{a)}$} & $\mathrm{F}$ & 60 & $\mathrm{OA}$ & 3 & Posterior & Gap imbalance & $4 \rightarrow 6 \rightarrow$ TKA \\
\hline & & & & 32 & Posterior & Gap imbalance & \\
\hline 9 & $\mathrm{M}$ & 80 & SONK & 102 & Anterior & Bearing wear & $4 \rightarrow 4$ \\
\hline 10 & $\mathrm{M}$ & 61 & $\mathrm{OA}$ & 65 & Anterior & Gap imbalance & $3 \rightarrow 5$ \\
\hline 11 & M & 76 & $\mathrm{OA}$ & 101 & Posterior & Impingement & $4 \rightarrow 5$ \\
\hline \multirow[t]{2}{*}{$12^{\text {a) }}$} & $\mathrm{F}$ & 50 & $\mathrm{OA}$ & 8 & Anterior & & \\
\hline & & & & 3 & Component malalignment & $3 \rightarrow 4 \rightarrow \mathrm{TKA}$ & \\
\hline \multirow[t]{2}{*}{$13^{\mathrm{a})}$} & $\mathrm{F}$ & 61 & $\mathrm{OA}$ & 69 & Anterior & MCL laxity & $6 \rightarrow 7 \rightarrow 7$ \\
\hline & & 62 & & 9 & Anterior & Acute trauma & \\
\hline \multirow[t]{2}{*}{$14^{\text {a) }}$} & $\mathrm{F}$ & 67 & $\mathrm{OA}$ & 15 & Anterior & Gap imbalance & $4 \rightarrow 5 \rightarrow \mathrm{TKA}$ \\
\hline & & 69 & & 28 & Anterior & MCL laxity & \\
\hline \multirow[t]{2}{*}{$15^{\text {a) }}$} & $\mathrm{F}$ & 79 & OA & 83 & Anterior \& posterior (breakage 2/3) & Bearing fracture & $4 \rightarrow 4 \rightarrow \mathrm{TKA}$ \\
\hline & & 84 & & 51 & Posterior & MCL laxity & \\
\hline \multirow[t]{3}{*}{$16^{\text {a) }}$} & $\mathrm{F}$ & 57 & OA & 1 & Anterior & Gap imbalance & $5 \rightarrow 7 \rightarrow 7 \rightarrow$ TKA \\
\hline & & 57 & & 1 & Anterior & Gap imbalance & \\
\hline & & 58 & & 10 & Anterior & Gap imbalance & \\
\hline \multirow[t]{2}{*}{$17^{\mathrm{a})}$} & $\mathrm{F}$ & 79 & OA & 42 & Medial & Gap imbalance & $4 \rightarrow 4 \rightarrow \mathrm{TKA}$ \\
\hline & & 81 & & 23 & Medial & Gap imbalance & \\
\hline \multirow[t]{2}{*}{$18^{\text {a) }}$} & $\mathrm{F}$ & 61 & OA & 31 & Anterior & MCL laxity & $4 \rightarrow 7 \rightarrow \mathrm{TKA}$ \\
\hline & & 62 & & 10 & Anterior & MCL laxity & \\
\hline
\end{tabular}

OA: osteoarthritis, TKA: total knee arthroplasty, SONK: spontaneous osteonecrosis of knee, MCL: medial collateral ligament.

a) Bearing sizes at exchange for the 1st, 2nd, and 3rd dislocations (if applicable) are presented in order. 

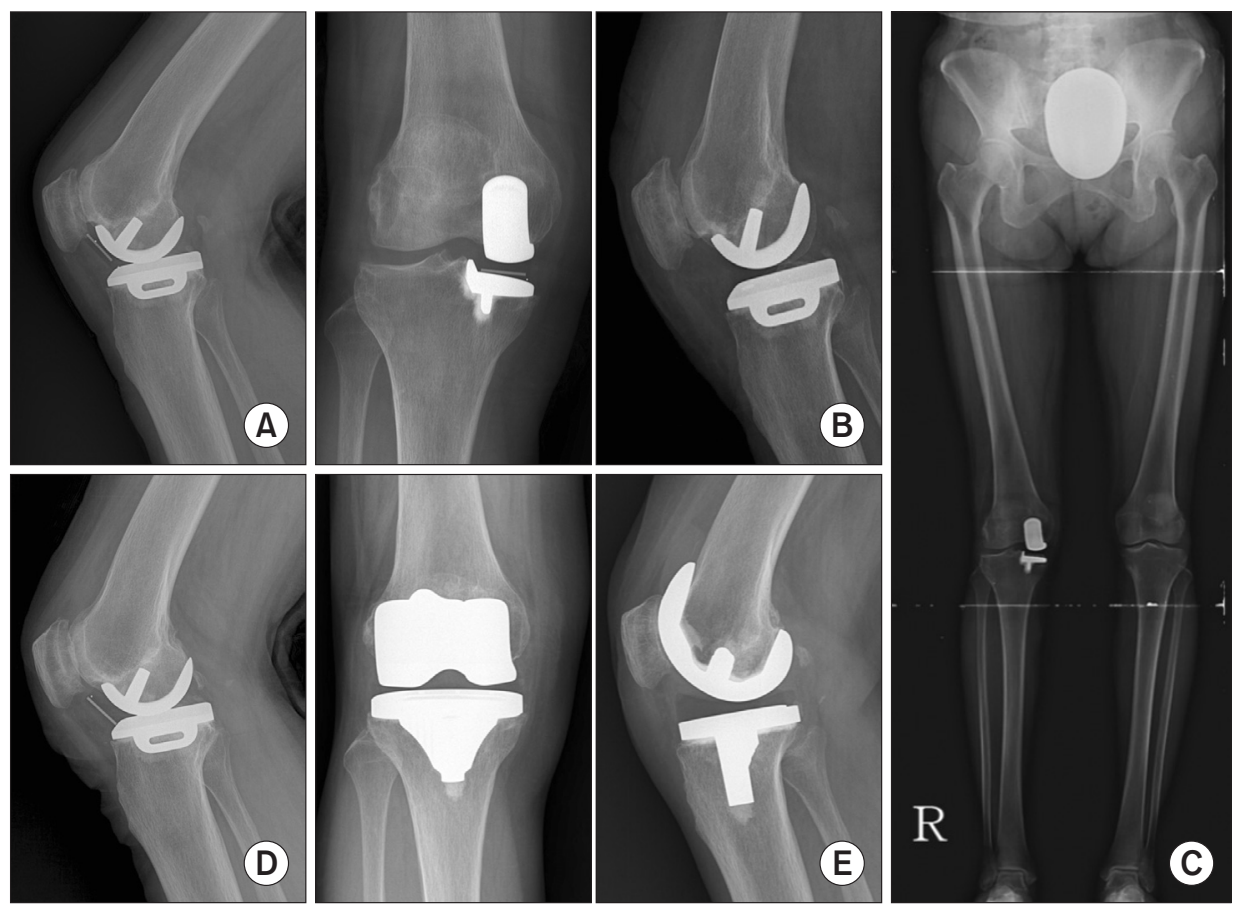

Fig. 1. A 61-year-old female patient underwent mobile medial unicompartmental knee arthroplasty (UKA) for medial compartmental osteoarthritis. (A) Anterior dislocation of mobile bearing occurred 31 months after medial UKA. (B) A 4-mm bearing was replaced with a $7-\mathrm{mm}$ bearing. (C) Valgus limb alignment was observed with use of the thicker bearing. (D) Recurrence of bearing dislocation was noted 10 months after bearing exchange. Intraoperatively, medial collateral ligament was found to be lax. (E) Conversion to total knee arthroplasty was performed.

Table 2. Comparison between the Non-Redislocation Group and the Redislocation Group

\begin{tabular}{|c|c|c|c|}
\hline Parameter & Non-redislocation group & Redislocation group & p-value \\
\hline No. of patients & 9 & 9 & \\
\hline Age (yr) & $66 \pm 10$ & $64 \pm 10$ & 0.960 \\
\hline Body mass index $\left(\mathrm{kg} / \mathrm{m}^{2}\right)$ & $25 \pm 7$ & $26 \pm 5$ & 0.213 \\
\hline \multicolumn{4}{|l|}{ Direction of 1st bearing dislocation } \\
\hline Posterior & 5 & 2 & 0.040 \\
\hline Medial & 0 & 1 & 0.250 \\
\hline Anterior \& posterior & 0 & 1 & 0.250 \\
\hline \multicolumn{4}{|l|}{ Bearing thickness (mm) } \\
\hline Index operation ${ }^{\text {a) }}$ & $3(3), 4(4), 6(2)$ & $3(2), 4(5), 5(1), 6(1)$ & 0.611 \\
\hline Preoperative mechanical FTA $\left({ }^{\circ}\right)^{\mathrm{c})}$ & $173.6 \pm 3.4$ & $173.9 \pm 2.0$ & 0.880 \\
\hline Postoperative mechanical FTA $\left({ }^{\circ}\right)^{\mathrm{c})}$ & $178.9 \pm 4.0$ & $179.2 \pm 1.5$ & 0.960 \\
\hline Femoral implant coronal alignment $\left(\left(^{\circ}\right)^{\mathrm{d})}\right.$ & $-0.8 \pm 2.8$ & $-1.3 \pm 5.2$ & 1 \\
\hline Femoral implant sagittal alignment $\left({ }^{\circ}\right)^{\mathrm{d})}$ & $5.6 \pm 7.6$ & $1.0 \pm 7.2$ & 0.238 \\
\hline Tibial implant coronal alignment $\left({ }^{\circ}\right)^{\mathrm{d})}$ & $1.2 \pm 2.3$ & $1.9 \pm 1.0$ & 0.275 \\
\hline Tibial implant sagittal alignment $\left({ }^{\circ}\right)^{\mathrm{d})}$ & $8.5 \pm 2.6$ & $7.6 \pm 2.8$ & 0.528 \\
\hline
\end{tabular}

Values are presented as mean \pm standard deviation or number.

FTA: femorotibial angle.

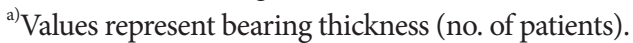

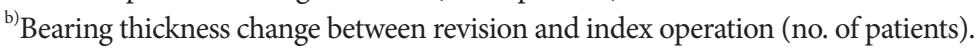

${ }^{c}$ A value of $<180^{\circ}$ means varus alignment; a value of $>180^{\circ}$ means valgus alignment.

${ }^{d)}$ Positive value means varus or flexion of implant; negative value means valgus or extension of implant. 


\section{Bearing Redislocation}

At a mean of 27 months (range, 1 to 90 months) after bearing exchange, 9 patients (50\%) had a recurrence of bearing dislocation. Five of nine patients $(60 \%)$ of these bearing redislocations occurred within 2 years after bearing exchange. Seven patients were re-revised to TKA, while 2 patients were treated with bearing exchange (Fig. 1). However, 1 patient suffered a third bearing dislocation 10 months after the second bearing exchange, so she underwent conversion to TKA. The other patient had no further dislocation of the bearing after the second bearing exchange at 8 years of follow-up. No changes were identified on the radiographic evaluation at the most recent follow-up.

Nine patients without bearing redislocation showed good to excellent clinical results following the bearing exchange at a mean follow-up of 55 months.

\section{Non-Redislocation Group vs. Redislocation Group}

There were no significant differences in pre-, intra-, and postoperative variables between the non-redislocation group and the redislocation group. We found that the non-redislocation group had a higher percentage of posterior dislocation of the bearing than the redislocation group ( $55.5 \%$ vs. $22.2 \%$, p=0.040) (Table 2 ). However, univariate logistic regression analysis showed no significant risk factors for bearing redislocation.

\section{Discussion}

The present study demonstrated a high rate of redislocation after bearing exchange for mobile bearing dislocation in medial UKA. Five of nine patients $(60 \%)$ of the bearing redislocations occurred within 2 years after bearing exchange, implying that the causes of bearing dislocations might not have been completely corrected during the revision operation. Even though bearing exchange is a simple and less invasive treatment option, our results suggest that bearing exchange alone should be carefully considered in selected patients having correctable causes such as impingement by remnant cement or bony spur, larger gap (thin bearing at the index operation), loss of entrapment by late bearing wear at the long-term follow-up, or acute trauma. In particular, when the cause of bearing dislocation cannot be identified or the causes of dislocation are irreparable intraoperatively (MCL laxity, serious component malalignment, component loosening, or serious flexion/extension gap mismatch) conversion to TKA should be considered.

A limited number of studies have been published on the outcomes or recurrence rate after isolated bearing exchange for mobile bearing dislocation in medial UKA. Kim et al. ${ }^{13)}$ reported that 24 mobile bearing dislocations in medial UKA were treated with bearing exchange. However, bearing redislocation occurred in 5 knees (21\%) after the treatment, which required conversion to TKA. Jung et al. ${ }^{17)}$ reported that the most common cause of a second re-operation after mobile bearing UKA was bearing dislocation in their cohort. In cases where patients underwent a third operation, $70 \%$ had bearing exchange with or without some minor procedure at the time of the second operation. All were converted to TKA at their third operation at a mean of only 7.7 months following their second operation. Our results are similar to those in previous studies in terms of the high rate of redislocation. However, as these results originated in the same country, it is uncertain whether similar results would be expected in other countries or populations.

When bearing dislocation occurs, the cause of dislocation should be determined before deciding a treatment method. If the underlying cause is correctable during the revision, a bearing exchange with the same or a slightly thicker size could be a preferred option after the cause has been addressed ${ }^{18)}$. In these cases, redislocation does not occur theoretically. However, underlying causes can be multifactorial. If the causes were a combination of correctable and irreparable factors, bearing redislocation is likely to occur even after a bearing exchange for which the correctable causes are addressed. In our study, two patients had bearing redislocation even after removal of the retained cement at the time of the first revision. There must have been other causes of bearing redislocation in these patients, which we might have ignored. When a definite cause of bearing dislocation is not identified, bearing exchange may not be the most proper treatment option; conversion to TKA would be a better option for definitive treatment.

The direction of bearing dislocation is suggestive of the cause, which may help us determine the treatment option when bearing dislocation occurs. In this study, 55.5\% of anterior dislocations led to bearing redislocation after bearing exchange, while two posterior dislocations were associated with bearing redislocation. It is difficult to explain why patients with anterior dislocation had a higher percentage of redislocation than those with posterior dislocation. Since the anterior rim of the bearing is higher than its posterior rim, posterior dislocation requires more distraction of the joint than anterior dislocation. Therefore, the displaced bearing is most commonly found in the anterior joint space, often in the suprapatellar pouch. Displacement into the posterior joint space suggests that the bearing has rotated $90^{\circ}$ (i.e., spinout) from which position it is as easy to dislocate backwards as 
it is to dislocate forwards, as the entrapment has decreased to $2 \mathrm{~mm}$. Based on our results, we suppose that bearing exchange using a thicker bearing may prevent spin-out due to an increase in entrapment, if there is no MCL insufficiency. In anterior dislocation without posterior impingement, a possible cause is gap imbalance, especially a narrow flexion or extension gap. As most cases of gap imbalance are irreparable during revision, a bearing exchange is likely to fail in this situation. Therefore, we recommend consideration of conversion to TKA in anterior dislocation without posterior impingement.

A bearing that is too thick may not guarantee effective treatment of a bearing dislocation. Due to fear of bearing redislocation, bearings that are two or three sizes greater in thickness than the original bearing are inserted in some patients. However, if a bearing that is two or three sizes thicker can be inserted into the space, MCL insufficiency should be suspected. Furthermore, overstuffing the space with a thicker bearing leads to stretching of the MCL and progression of MCL laxity ${ }^{19)}$. Consequently, such a thicker bearing does not provide stability but instead increases the risk of bearing redislocation. Increased strain of the MCL also causes unexpected pain, limited range of motion, and instabil$i^{20)}$. In our cases, 1 patient had valgus limb alignment after the exchange for a bearing three sizes greater in thickness for the treatment of bearing dislocation. One year later, the patient had a second bearing dislocation. Intraoperatively, we found that the MCL was insufficient, and thus conversion to TKA was performed.

The clinical outcomes of the 9 patients without redislocation following bearing exchange are comparable to those of the patients without bearing dislocation. Therefore, if the patient is properly selected, a bearing exchange should be the best treatment option for bearing dislocation. However, a thicker bearing exchange results in more valgus mechanical limb alignment ${ }^{21)}$. While it is uncertain whether valgus mechanical alignment affects clinical outcomes and long-term survivorship in the future, postoperative valgus mechanical alignment is not generally recommended due to the likely progression of arthritis in the lateral compartment. In addition, previous studies have suggested that a few degrees of varus alignment provides better results ${ }^{22,23)}$. In our study, lateral compartment arthritis was not seen in any patients at a mean follow-up of 55 months. However, long-term followup should be performed to see if lateral compartment arthritis develops.

There are several limitations of this study. First, the retrospective study design and the small number of patients from a single institution made it difficult to determine the exact causes of bear- ing dislocation. Second, the surgeon's preference for bearing exchange might have affected the percentage of bearing dislocation. Third, our results may not be applicable to other populations. Fourth, it remains to be seen whether the 9 patients without bearing redislocation will survive at longer term follow-up. Despite these limitations, we believe that the present study provides clinicians with useful information for choosing a treatment method for mobile bearing dislocation in medial UKA.

\section{Conclusions}

This study showed a high rate of bearing redislocation after isolated, mobile bearing exchange for bearing dislocation following medial UKA. Therefore, bearing exchange as a sole treatment should be carefully considered in selected patients with correctable causes. If the cause of bearing dislocation cannot be determined or corrected during revision, conversion to TKA may be a better option for definitive treatment.

\section{Conflict of Interest}

No potential conflict of interest relevant to this article was reported.

\section{References}

1. Pandit H, Hamilton TW, Jenkins C, Mellon SJ, Dodd CA, Murray DW. The clinical outcome of minimally invasive Phase 3 Oxford unicompartmental knee arthroplasty: a 15year follow-up of 1000 UKAs. Bone Joint J. 2015;97:1493500.

2. Yoshida K, Tada M, Yoshida H, Takei S, Fukuoka S, Nakamura H. Oxford phase 3 unicompartmental knee arthroplasty in Japan: clinical results in greater than one thousand cases over ten years. J Arthroplasty. 2013;28(9 Suppl):168-71.

3. Lee SY, Bae JH, Kim JG, Jang KM, Shon WY, Kim KW, Lim HC. The influence of surgical factors on dislocation of the meniscal bearing after Oxford medial unicompartmental knee replacement: a case-control study. Bone Joint J. 2014; 96:914-22.

4. Emerson RH, Alnachoukati O, Barrington J, Ennin K. The results of Oxford unicompartmental knee arthroplasty in the United States: a mean ten-year survival analysis. Bone Joint J. 2016;98(10 Supple B):34-40.

5. Choy WS, Kim KJ, Lee SK, Yang DS, Lee NK. Mid-term results of oxford medial unicompartmental knee arthroplasty. 
Clin Orthop Surg. 2011;3:178-83.

6. Goodfellow J, O'Connor J, Pandit H, Dodd C, Murray D. Unicompartmental arthroplasty with the oxford knee. 2nd ed. Oxford: Goodfellow Publishers Ltd: 2015.

7. Hang JR, Stanford TE, Graves SE, Davidson DC, de Steiger RN, Miller LN. Outcome of revision of unicompartmental knee replacement. Acta Orthop. 2010;81:95-8.

8. van Tienen TG, Taylor SJ, Brink RB. Successful salvage of a recurrently dislocating Oxford medial unicompartmental bearing. J Arthroplasty. 2010;25:497.

9. Gul SF, Davies AP. Successful revision of polyethylene only, after delayed presentation of a dislocated bearing in an Oxford unicompartmental knee replacement. BMJ Case Rep. 2013;2013.

10. Fujii T, Matsui Y, Noboru M, Inagaki Y, Kadoya Y, Tanaka Y. Meniscal Bearing Dislocation of Unicompartmental Knee Arthroplasty with Faint Symptom. Case Rep Orthop. 2015; 2015:217842.

11. Tibrewal S, Pandit H, McLardy-Smith P, Tibrewal SB, Murray DW. Posterior dislocation of the Oxford knee meniscal bearing: a treatment option. J Orthop Traumatol. 2014;15: 59-62.

12. Burnett RS, Nair R, Hall CA, Jacks DA, Pugh L, McAllister MM. Results of the Oxford Phase 3 mobile bearing medial unicompartmental knee arthroplasty from an independent center: 467 knees at a mean 6-year follow-up: analysis of predictors of failure. J Arthroplasty. 2014;29(9 Suppl):193-200.

13. Kim KT, Lee S, Lee JI, Kim JW. Analysis and Treatment of Complications after Unicompartmental Knee Arthroplasty. Knee Surg Relat Res. 2016;28:46-54.

14. Anciano Granadillo JR, Worland RL, Canellas Trobat A. Recurrent mobile bearing dislocation after minimally invasive unicompartmental knee arthroplasty. Med Balear 2011;26: 32-5.

15. Murray DW, Goodfellow J, O'Connor, Dood C. Oxford ${ }^{\mathrm{m}}$ unicompartmental knee manual of the surgical technique [Internet]. Bridgend: Biomet UK Ltd; 1999 [cited $2018 \mathrm{Feb}$ 11]. Available from: http://www.biomet.se/resource/17723/ Oxford\%20ST.pdf.

16. Murray DW, Fitzpatrick R, Rogers K, Pandit H, Beard DJ, Carr AJ, Dawson J. The use of the Oxford hip and knee scores. J Bone Joint Surg Br. 2007;89:1010-4.

17. Jung KA, Kumar R, Lee SC, Ahn HS, Gondalia V, Ong AC, Park HY. Causes and distribution of a re-operation after unicompartmental knee arthroplasty. Orthop Proc. 2016; 98B(Supp 8):70.

18. Thienpont E. Conversion of a unicompartmental knee arthroplasty to a total knee arthroplasty: can we achieve a primary result? Bone Joint J. 2017;99(1 Supple A):65-9.

19. Imhauser MKC, Warth L, Lipman J, Westrich GH, Cross MB, Mayman DJ, Pearle A, Wright T. Tibial polyethylene thickness affects medial compartment translation and ligament loads in unicompartmental knee arthroplasty. Orthop Proc. 2016;98B(Supp 10):135.

20. Heyse TJ, El-Zayat BF, De Corte R, Scheys L, Chevalier Y, Fuchs-Winkelmann S, Labey L. Balancing UKA: overstuffing leads to high medial collateral ligament strains. Knee Surg Sports Traumatol Arthrosc. 2016;24:3218-28.

21. Kim SJ, Bae JH, Lim HC. Factors affecting the postoperative limb alignment and clinical outcome after Oxford unicompartmental knee arthroplasty. J Arthroplasty. 2012;27:12105.

22. Zuiderbaan HA, van der List JP, Chawla H, Khamaisy S, Thein R, Pearle AD. Predictors of subjective outcome after medial unicompartmental knee arthroplasty. J Arthroplasty. 2016;31:1453-8.

23. Vasso M, Del Regno C, D'Amelio A, Viggiano D, Corona K, Schiavone Panni A. Minor varus alignment provides better results than neutral alignment in medial UKA. Knee. 2015; 22:117-21. 